\section{D) Check for updates}

Cite this: Org. Biomol. Chem., 2020, 18, 8109

\title{
Total synthesis of the actinoallolides and a designed photoaffinity probe for target identification $\uparrow$
}

\begin{abstract}
Matthew J. Anketell, (D) Theodore M. Sharrock and lan Paterson (D)*
The actinoallolides are a family of polyketide natural products isolated from the bacterium Actinoallomurus fulvus. They show potent biological activity against trypanosomes, the causative agents of the neglected tropical diseases human African trypanosomiasis (sleeping sickness) and Chagas disease, while exhibiting no cytotoxicity against human cell lines. Herein, we give a full account of our strategy evolution towards the synthesis of this structurally unique class of 12-membered macrolides, which culminated in the first total synthesis of $(+)$-actinoallolide $A$ in 20 steps and $8 \%$ overall yield. Subsequent latestage diversification then provided ready access to the congeneric (+)-actinoallolides B-E. Enabled by this flexible and efficient endgame sequence, we also describe the design and synthesis of a photoaffinity probe based on actinoallolide A to investigate its biological mode of action. This will allow ongoing labelling studies to identify their protein binding target(s).
\end{abstract}

Received 4th September 2020, Accepted 25th September 2020 DOI: $10.1039 / \mathrm{d0ob01831g}$ rsc.li/obc
Fortunately, the natural world possesses a rich and structurally diverse source of antiparasitic compounds. ${ }^{17}$ The actinoallolides (Fig. 1), a family of novel polyketides, are one such example, isolated by Iwatsuki and co-workers from a cultured strain (MK10-036) of the actinomycete bacterium Actinoallomurus fulvus, obtained from the roots of Capsicum neglected tropical diseases human African trypanosomiasis (sleeping sickness) ${ }^{3-5}$ and Chagas disease, ${ }^{6-10}$ both caused by protozoan parasites of the genus Trypanosoma. These illnesses are estimated to account for a loss of 455000 disabilityadjusted life years annually. ${ }^{11}$ Trypanosomes are extremely adept at evading the immune system, and these neglected tropical diseases can only be cleared from the body with the help of chemotherapeutic agents. ${ }^{12}$ However, the ongoing development of effective pharmaceutical agents against the causative agents of these diseases has been challenging due to two major factors. ${ }^{13,14}$ First, the eukaryotic nature of protozoan parasites means that trypanosomes are biologically more closely related than bacteria to human cells. This renders the development of selective anti-trypanosomal drugs more challenging. ${ }^{15}$ Secondly, as these diseases mainly affect less economically developed countries, a lack of financial incentive to develop new drugs has led to an urgent need for better, more effective and safer treatments for these neglected diseases. ${ }^{16}$

University Chemical Laboratory, University of Cambridge, Lensfield Road, CB2 1EW, UK

$\dagger$ Electronic supplementary information (ESI) available: Full experimental details and characterisation of new compounds, together with NMR comparisons of the synthetic and natural actinoallolides. See DOI: 10.1039/D0OB01831G
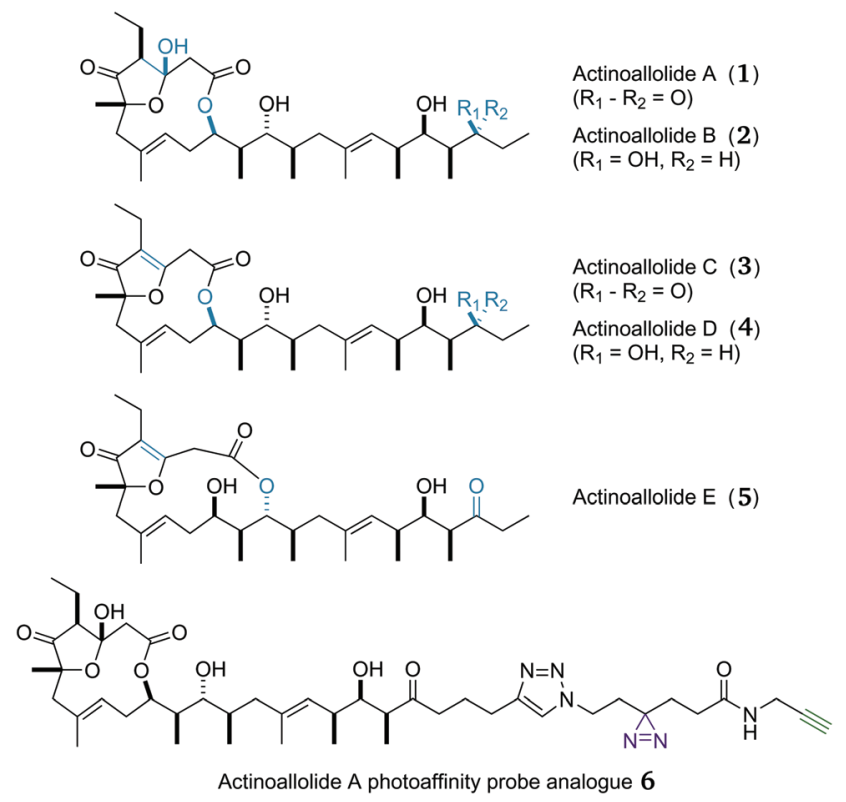

Fig. $13 D$ structures of the five actinoallolide congeners 1-5 and the designed photoaffinity probe analogue 6 . 
fruitescens collected in Thailand. ${ }^{18}$ The 3D structure of actinoallolide A (1) was determined by spectroscopic analysis and $\mathrm{X}$-ray crystallography, which revealed the presence of 10 stereocentres, two trisubstituted alkenes and a 12-membered macrolactone incorporating a five-membered hemiacetal. In addition, the structures of actinoallolides B-E (2-5) were confirmed by chemical correlation with actinoallolide A.

The actinoallolides were tested against three strains of Trypanosoma and compared with several commonly used antitrypanosomal drugs. Actinoallolides A-E were also tested for biological activity against Trypanosoma brucei brucei, the strain responsible for animal trypanosomiasis (Nagana disease) and compared with three of the most commonly used drug treatments. They all compared favourably, with actinoallolide A determined as the most potent by two orders of magnitude, with an $\mathrm{IC}_{50}$ of $8.3 \mathrm{nM}$. Actinoallolide A (1) was also tested against Trypanosoma brucei rhodesiense, a strain responsible for human African trypanosomiasis and Trypanosoma cruzi, the causative agent of Chagas disease, showing double the potency of benznidazole. Actinoallolide A (1) showed no observable activity against MRC-5 human cells, giving it a high selectivity index $(>20000) \cdot{ }^{19}$ It was inactive against Gram-positive and negative bacteria and against yeast and fungi, indicating a highly specific mechanism of antitrypanosomal activity that is yet to be determined.

The isolation group have also identified the actinoallolide biosynthetic gene cluster, proposed a biosynthesis, ${ }^{20}$ and reported preliminary synthetic studies. ${ }^{21}$ Herein, we give a full account of the first total synthesis of the actinoallolides, ${ }^{22}$ which was enabled by a challenging ring-closing metathesis reaction to form the macrocyclic trisubstituted alkene, the most complex example of its kind. ${ }^{23}$ As the biological target and mechanism of action of the actinoallolides are unknown, we sought to leverage our total synthesis to shed light on these unanswered questions. Based on a highly efficient endgame, we now report the design and synthesis of the actinoallolide A-based probe 6 for target identification using photoaffinity labelling. ${ }^{24,25}$

\section{Results and discussion}

\section{Retrosynthetic analysis of actinoallolide A}

Our proposed key bond disconnections (Scheme 1) were of the $\mathrm{C}_{1}$ ester and the $\mathrm{C}_{8}-\mathrm{C}_{9}$ alkene to form two fragments of roughly equal size and complexity: the macrocycle precursor 7 and the side chain 8. A major advantage of this approach is that it allows a flexible fragment coupling strategy whereby the two coupling steps could be undertaken in either order; cross metathesis could be followed by macrolactonisation, or esterification could be followed by ring-closing metathesis as desired.

The $\mathrm{C}_{1}-\mathrm{C}_{8}$ fragment 7 would be forged from natural $(S)$ lactic acid with key bonds formed via a Seebach alkylation and a diastereoselective aldol reaction. Protection of the $\mathrm{C}_{3}$ and $\mathrm{C}_{6}$ alcohols would allow late-stage unmasking of the delicate five-

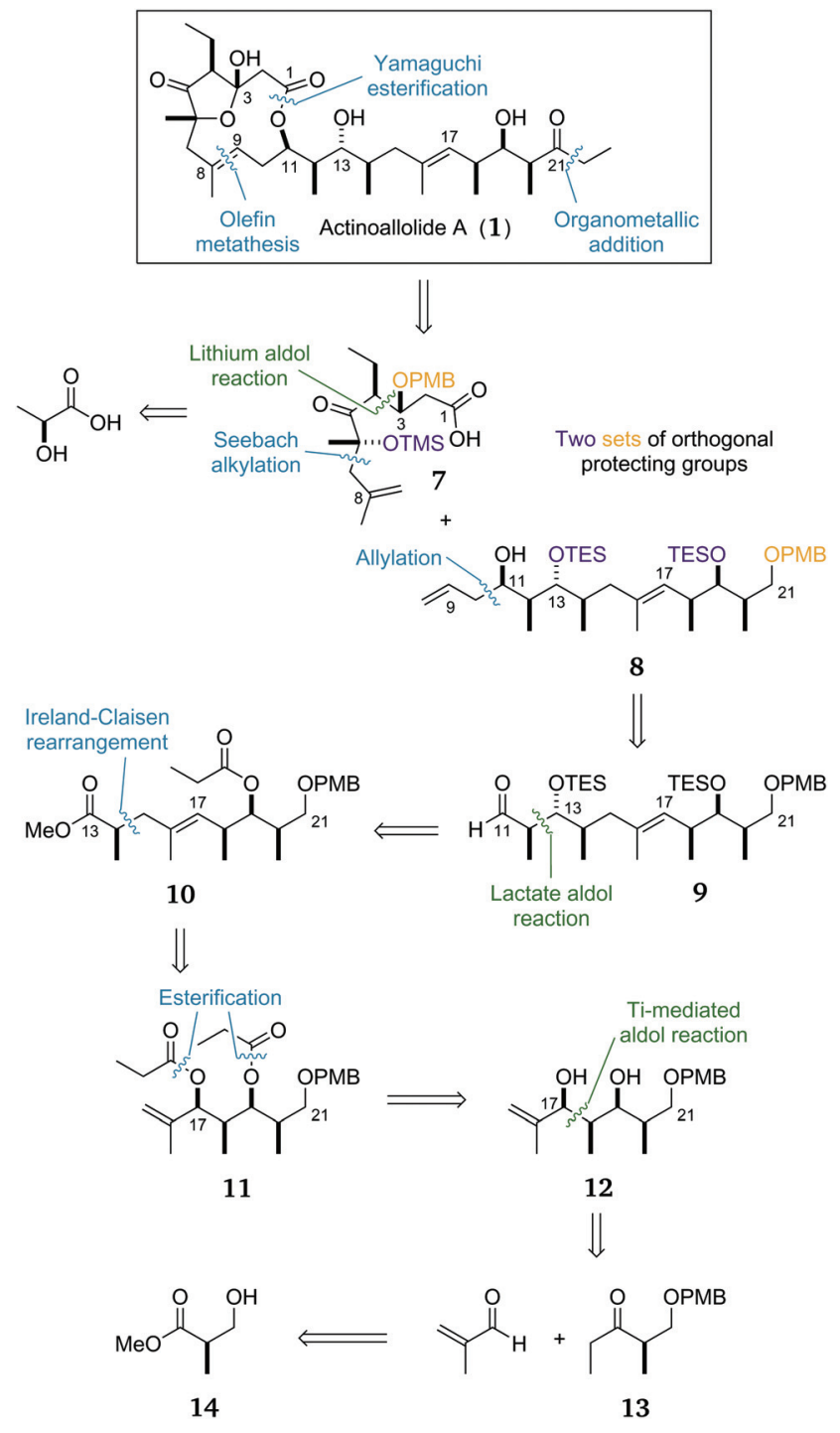

Scheme 1 Initially proposed retrosynthesis of actinoallolide A with the three key aldol reactions highlighted in green.

membered hemiacetal after deprotection and oxidation at $\mathrm{C}_{3}$ to give actinoallolide $\mathrm{A}$.

The side chain contains two distinct stereoclusters. The $\mathrm{C}_{11}-\mathrm{C}_{14}$ stereotetrad was envisaged to be constructed by a substrate-controlled allylation of $\mathbf{9}$ preceded by a lactate aldol reaction to reveal, after redox adjustment, ester 10 . The isolated $\mathrm{C}_{14}$ stereocentre in $\mathbf{1 0}$ could then be installed by an Ireland-Claisen rearrangement from the diester 11. The 'all syn' stereotetrad in $\mathbf{1 2}$ can then be formed by a titaniummediated aldol reaction/in situ reduction between methacrolein and ethyl ketone 13, itself synthesised in three steps from $(R)$-Roche ester (14). The decision to attach the terminal ethyl group at such a late stage was made in order to form the $\mathrm{C}_{21}$ ketone as late as possible to facilitate late-stage diversification. A more complex organometallic reagent could be used to add any modified tail in place of the ethyl group. 
Finally, the decision to utilise two sets of orthogonal protecting groups was informed by our planned endgame. The hydroxyl groups at $\mathrm{C}_{21}$ and $\mathrm{C}_{3}$ require oxidation, whereas those at $\mathrm{C}_{6}, \mathrm{C}_{13}$ and $\mathrm{C}_{19}$ do not. Thus it was planned to use benzyl ether-based groups for the former and silyl ethers for the latter hydroxyl groups.

\section{Synthesis of the $\mathrm{C}_{9}-\mathrm{C}_{21}$ side chain fragment (8)}

The synthesis of side chain fragment $\mathbf{8}$ commenced with ethyl ketone 13. ${ }^{26}$ In previous work, the 'all-syn' aldol selectivity required for the construction of $\mathbf{1 2}$ had been achieved by use of tin(II) triflate as a Lewis acid. ${ }^{27,28}$ While effective, the use of tin(II) triflate was procedurally cumbersome, especially on scale owing to its capricious preparation. We sought to improve upon this process by combining two recent developments in aldol chemistry. Romea and Urpí have reported the use of titanium Lewis acids for the highly selective aldol reactions of protected $\beta$-hydroxy ketones ${ }^{29}$ and $\alpha$-hydroxy ketones with an in situ reduction to afford the syn-1,3-diol. ${ }^{30}$ It was reasoned that combining these two processes would afford the desired 'all-syn' stereotetrad in diol 12. In the event, application of this in situ reduction with $\mathrm{LiBH}_{4}$ to the aldol addition of ketone 13 and methacrolein provided diol 12 in 90\% yield as a single diastereomer (Scheme 2). Owing to the oxophilicity of the titanium and boron complexes formed in the reaction, an extended workup incorporating treatment with hydrogen peroxide and multiple washings with Rochelle salt solution was found to be necessary for release of $\mathbf{1 2}$.

After selective monoesterification of the allylic alcohol in diol 12 was found to be problematic, it was decided to proceed through the required Ireland-Claisen rearrangement ${ }^{31}$ with diester 11. Only the $\mathrm{O}_{17}$ ester was correctly positioned to undergo the $[3,3]$-sigmatropic rearrangement while the $\mathrm{O}_{19}$ ester would be removed during the later reduction of the $\mathrm{C}_{13}$ methyl ester to the aldehyde. Guided by a similar Ireland-
Claisen rearrangement used in the total synthesis of ebelactone, ${ }^{32}$ we began investigating this key step. Initial attempts at performing this reaction led to appreciable formation of the $\mathrm{C}_{14} \alpha$-silylated by-product. This TMS group could be removed with $\mathrm{K}_{2} \mathrm{CO}_{3}$ in $\mathrm{MeOH}$ but, as would be expected, led to significant epimerisation. The epimer was visible by NMR and provided evidence that the Ireland-Claisen product had been obtained as a single diastereomer. This side reaction is proposed to occur via enolisation of the formed silyl ester by excess LDA and trapping of this enolate with TMSCl. As such, it was important to precisely control the equivalents of LDA used. In order to avoid quenching any LDA, it is necessary to remove all traces of $\mathrm{HCl}$ from the large excess of TMSCl used. In the ebelactone work, this was accomplished by premixing TMSCl with $\mathrm{Et}_{3} \mathrm{~N}$ and centrifuging the resulting suspension to remove the precipitate. In this work, removal of the precipitate was more conveniently accomplished via filtration through a syringe tip filter (PVDF membrane, $0.45 \mu \mathrm{m}$ pores). Optimising the quantity of LDA used increased the yield to $73 \%$ with no observable formation of the $\alpha$-silylated by-product. For ease of isolation, the carboxylic acid product was methylated without purification to afford methyl ester $\mathbf{1 0 .}$

Pleasingly, the DIBAL-H reaction of this ester $\mathbf{1 0}$ proceeded well, reducing the methyl ester at $\mathrm{C}_{13}$ to the corresponding aldehyde and liberating the free hydroxyl group at $\mathrm{C}_{19}$, setting the stage for the second strategic aldol reaction. While substrate control was used to construct all other stereocentres in side chain fragment $\mathbf{8}$, the anticipated mismatch between the Felkin-Anh selectivity of aldehyde $\mathbf{1 5}$ and the desired configuration at the $\mathrm{C}_{13}$ hydroxyl stereocentre made it impossible in this case. Using boron aldol methodology developed in the group,$^{33,34}$ we were able to couple aldehyde 15 with lactatederived ketone $\mathbf{1 6}$ to form anti adduct $\mathbf{1 7}$ as a single diastereomer, overriding the inherent facial selectivity of aldehyde $\mathbf{1 5}$ to install the $\mathrm{C}_{12}$ and $\mathrm{C}_{13}$ stereocentres. Protection of both

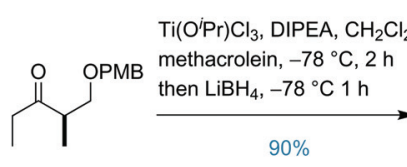

13

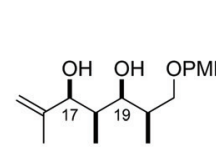

12

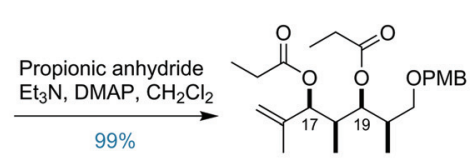

11

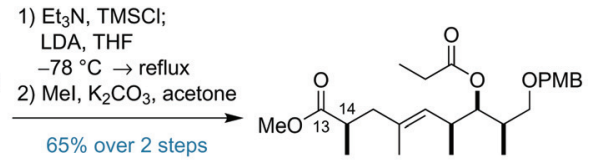

10 DIBAL-H, PhMe $-78^{\circ} \mathrm{C}, 1 \mathrm{~h}$ $\checkmark 85 \%$

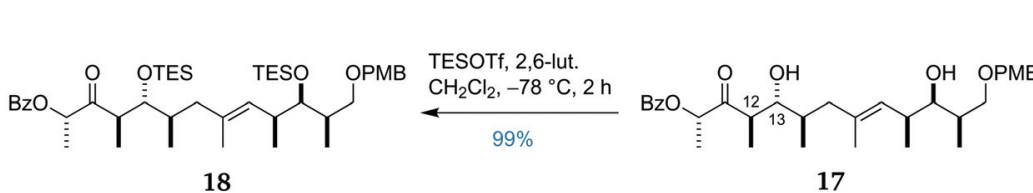

1) $\mathrm{LiBH}_{4}, \mathrm{THF}, \mathrm{rt}, 18 \mathrm{~h}$ 2) $\mathrm{NaIO}_{4} / \mathrm{SiO}_{2}, \mathrm{CH}_{2} \mathrm{Cl}_{2} \mathrm{rt}, 1 \mathrm{~h}$ $80 \%$ over 2 steps

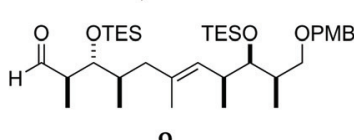

9

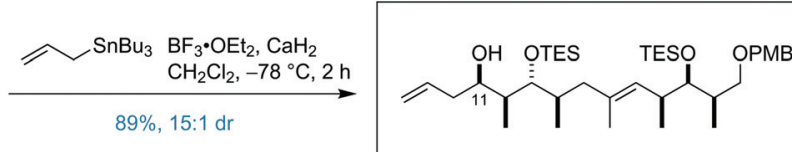

Side chain fragment $\mathbf{8}$ 3 Steps LLS, $21 \%, 2.3 \mathrm{~g}$ scale

Scheme 2 Synthesis of the side chain fragment 8. 
hydroxyl groups as TES ethers then afforded 18 which underwent a standard two-step auxiliary removal procedure to provide aldehyde $9 .{ }^{33}$ Addition of the allyl moiety to 9 to complete the side chain fragment $\mathbf{8}$ required some optimisation with the use of allylmagnesium chloride or allyltrimethylsilane leading to low diastereoselectivity. Fortunately, it was found that the utilisation of allyltributylstannane in a HosomiSakurai reaction ${ }^{35,36}$ with the non-chelating Lewis acid $\mathrm{BF}_{3} \cdot \mathrm{OEt}_{2}$ afforded 8 in high yield with $15: 1 \mathrm{dr}$.

This completed the scalable and high-yielding synthesis of the side chain fragment $\mathbf{8}$ in 13 linear steps from $(R)$-Roche ester (14) with an overall yield of $21 \%$. This excellent yield (an average of $89 \%$ per step) and the scalability of the route allowed $2.3 \mathrm{~g}$ to be prepared, providing ample material for development of the endgame.

\section{Synthesis of the $\mathrm{C}_{1}-\mathrm{C}_{8}$ macrocycle precursor fragment}

The synthesis of the macrocycle precursor fragment began with the construction of dioxolanone 19 via a two-step procedure (Scheme 3), ${ }^{37}$ allowing an enolate alkylation ${ }^{38}$ with methallyl bromide to install the $\mathrm{C}_{6}$ stereocentre. This reaction proved difficult to optimise, with a $53 \%$ yield of 20 , albeit as a single diastereomer. This was due to formation of a major byproduct due to self-reaction of the dioxolanone. ${ }^{39}$ However, the modest yield was deemed sufficient and allowed the preparation of 20 on a multi-gram scale.

The removal of the pivaldehyde-derived auxiliary via ring opening of dioxolanone 20 was the next task to be accomplished. In preliminary studies, the desired conversion to propyl ketone 22 was achieved using a four-step sequence commencing with ring opening to the methyl ester. We envisaged abridging this sequence by using a different ring-opening pro- cedure. The first attempt to convert the dioxolanone to the propyl ketone directly by addition of propylmagnesium bromide, however, was unsuccessful, yielding either unreacted starting material or the double addition product. Ring opening to the Weinreb amide 21 was trialled next and proceeded in excellent yield using the in situ generated anion of $\mathrm{N}, \mathrm{O}$-dimethylhydroxylamine. With this result, a one-pot conversion to the desired propyl ketone was examined. However, the conversion of $\mathbf{2 1}$ to the corresponding propyl ketone in situ proved unsuccessful even with warming to room temperature. Weinreb amide $\mathbf{2 1}$ was next silyl protected and converted to propyl ketone 22 , required for the planned $\mathrm{C}_{3}-\mathrm{C}_{4}$ bondforming lithium-mediated aldol reaction. As the protecting group strategy utilised a silyl group at $\mathrm{C}_{6}$ and a PMB group at $\mathrm{C}_{3}$, the $\mathrm{C}_{1}$ protecting group was required to be orthogonal to both.

From propyl ketone 22, two routes to macrocycle precursor fragment 7 were initially considered. The first plan was to utilise an orthoester protecting group on aldehyde 23 , as this would allow access to $\mathrm{C}_{1}$ at the desired carboxylic acid oxidation level. This lithium-mediated aldol reaction afforded syn adduct 24, providing the desired diastereomer in good yield and with useful diastereoselectivity. We had planned to protect the $\mathrm{C}_{3}$ alcohol with a PMB group, however all attempted conditions led to no reaction or to degradation, presumably owing to steric congestion. Furthermore, removal of the orthoester protecting group could not be achieved without degradation due to the acid and base sensitivity of the aldol adduct.

With this approach proving to be unviable, $\mathrm{C}_{1}$ was next introduced at the protected alcohol oxidation state. Noting that PMB groups can be oxidatively transposed, ${ }^{40}$ this second plan was to utilise a PMB group on aldehyde 25 to produce

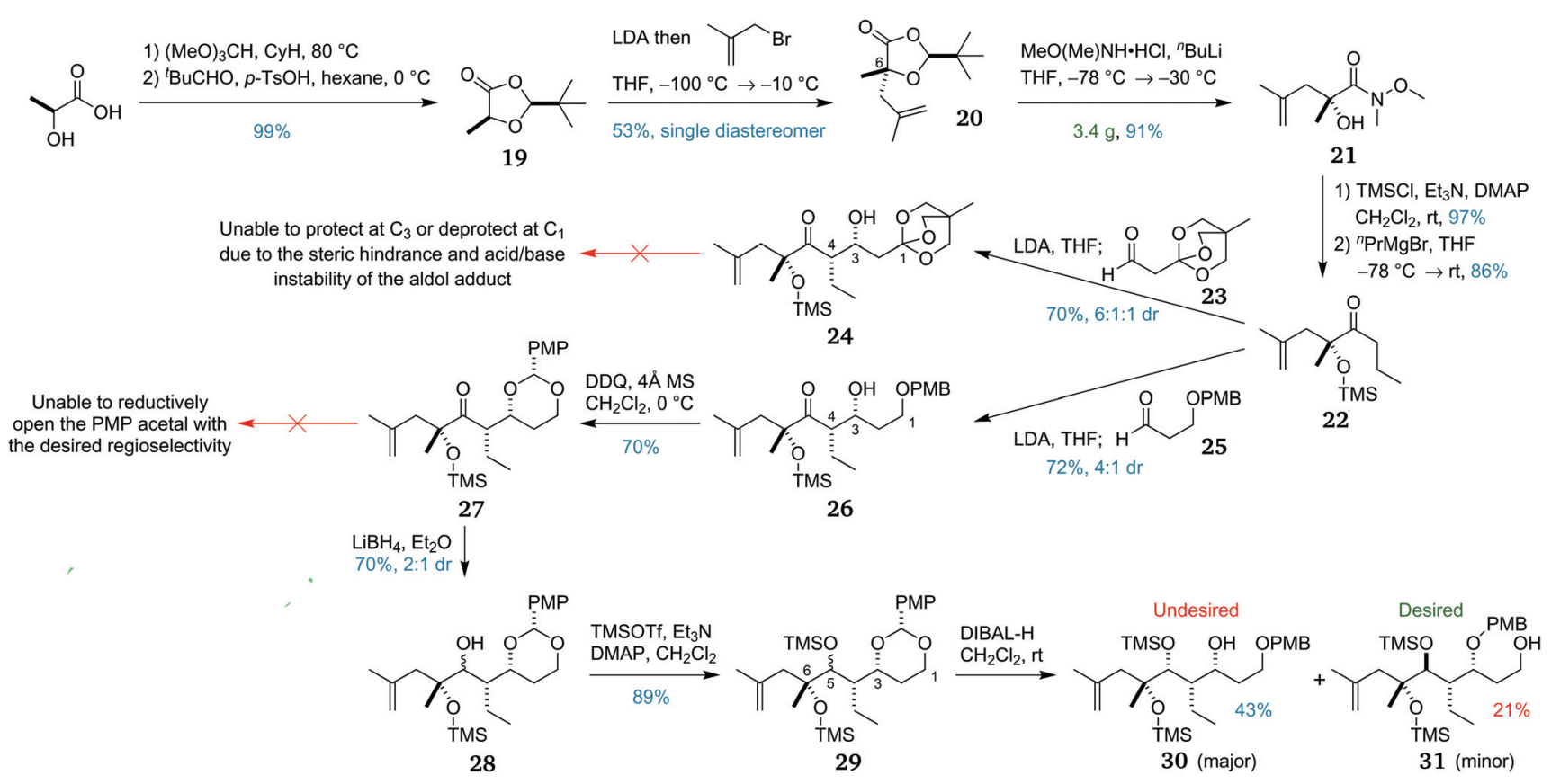

Scheme 3 Synthesis of the key propyl ketone 22 and attempts at elaboration. 
aldol adduct 26. As before, the lithium-mediated aldol reaction afforded the syn adduct in good yield, in a $4: 1$ ratio of diastereomers. The major diastereomer was then converted to PMP acetal 27 by treatment with DDQ under anhydrous conditions. Disappointingly, subsequent attempts at reductive PMP opening to the primary alcohol were all unsuccessful, with the use of DIBAL- $\mathrm{H}^{40}$ or $\mathrm{NaCNBH}_{3} / \mathrm{TMSCl}^{41}$ causing side-reactions or reacting with the undesired regioselectivity to revert to secondary alcohol 26. This unexpected selectivity may be due to preferential chelation of the Lewis acid between the $\mathrm{C}_{3}$ oxygen and the ketone rather than the less hindered $\mathrm{C}_{1}$ oxygen as would normally be expected.

Given that this selectivity problem was likely due to the propensity of the $\mathrm{C}_{5}$ ketone to coordinate with the adjacent $\mathrm{C}_{3}$ oxygen, it was proposed that reducing the $\mathrm{C}_{5}$ ketone and transiently protecting the resulting alcohol as a TMS ether might circumvent this impasse. Ketone 27 was thus reduced using $\mathrm{LiBH}_{4}$ to form secondary alcohol 28 in 70\% yield and 2:1 dr. The diastereomers were inseparable by chromatography so the alcohols were then silylated to form 29. This mixture of diastereomeric PMP acetals then underwent reductive opening with DIBAL-H. Surprisingly, the two epimeric acetals reacted with opposing regioselectivities, with the major diastereomer forming the undesired secondary alcohol $\mathbf{3 0}$ and the minor diastereomer forming the desired primary alcohol $\mathbf{3 1}$.

In light of this mixed result, it was reasoned that if we could override the inherent substrate selectivity of ketone 27 to reduction, and obtain the 1,3-anti diastereomer selectively, this might provide a viable route to the macrocycle fragment. Testing a range of simple reducing agents was fruitless, with each one either giving the undesired selectivity or no reaction. It was next decided to attempt the reduction of the $\mathrm{C}_{5}$ ketone prior to PMP acetal formation, using the $\mathrm{C}_{3}$ alcohol in 26 to direct a 1,3-anti Evans-Tishchenko ${ }^{42}$ or Evans-Saksena reduction. ${ }^{43}$ With prolonged reaction time and an excess of the samarium catalyst, ${ }^{42}$ it was possible to achieve a low yield of $10 \%$ with $2: 1 \mathrm{dr}$ for the former reaction, but no product was obtained under the latter conditions. This was attributed to the steric hindrance of the $\mathrm{C}_{5}$ ketone with its quaternary $\alpha$ stereocentre incorporating a bulky OTMS group.
These frustrations en route to the macrocycle precursor fragment led us to consider more substantial modifications to the route. The efforts thus far had focused on installing a PMB protecting group at $\mathrm{C}_{3}$, either by a 'PMB transposition'of the $\mathrm{PMB}$ group at $\mathrm{C}_{1}$, or by direct protection of the alcohol at $\mathrm{C}_{3}$. The former method had failed due to the selectivity problems with opening the intermediate PMP acetal and the latter had failed due to the steric hindrance of aldol adduct $\mathbf{2 6}$ and its instability under forcing conditions. To circumvent these problems within the constraints of the overall protecting group strategy - that is to allow for the chemoselective oxidative removal of the $\mathrm{C}_{3}$ and $\mathrm{C}_{21}$ protecting groups in one operation, it was proposed to switch the $\mathrm{C}_{3}$ protecting group from PMB to PMBM (para-methoxybenzyloxymethyl). ${ }^{44}$ Like the PMB group, it can be oxidatively removed orthogonally to silyl groups but can be installed under far milder conditions. However, as the PMBM group was liable to cleavage under oxidative conditions, this alteration necessitated a revision of the $\mathrm{C}_{1}$ protecting group such that it could be removed orthogonally to the PMBM group. Given that the aromatic ring in the DMB (3,4dimethoxybenzyl ${ }^{45}$ group is more electron rich than in the PMBM group, it was anticipated that selective oxidative cleavage of the DMB group would be possible. ${ }^{46}$

The corresponding DMB-protected aldehyde 32 required for the aldol coupling was prepared via a three-step procedure. ${ }^{47}$ This aldehyde then underwent an analogous lithium-mediated aldol reaction with propyl ketone 22, providing syn adduct 33 (Scheme 4) in similar yield and dr to that obtained from the PMB-protected variant. This was subsequently PMBM protected using the mild base DIPEA in refluxing acetonitrile overnight to afford PMBM ether 34 in excellent yield. With triplyprotected aldol adduct $\mathbf{3 4}$ in hand, selective DMB cleavage was next attempted. Fortunately, one equivalent of DDQ with careful monitoring afforded primary alcohol 35 in excellent yield, with complete retention of the PMBM group. This then underwent a double oxidation sequence; first was a Swern oxidation to give aldehyde 36 , then a Pinnick oxidation provided the complete macrocycle precursor fragment 37.

Overall, macrocycle precursor fragment 37 was synthesised in 10 linear steps from $(S)$-lactic acid with an overall yield of
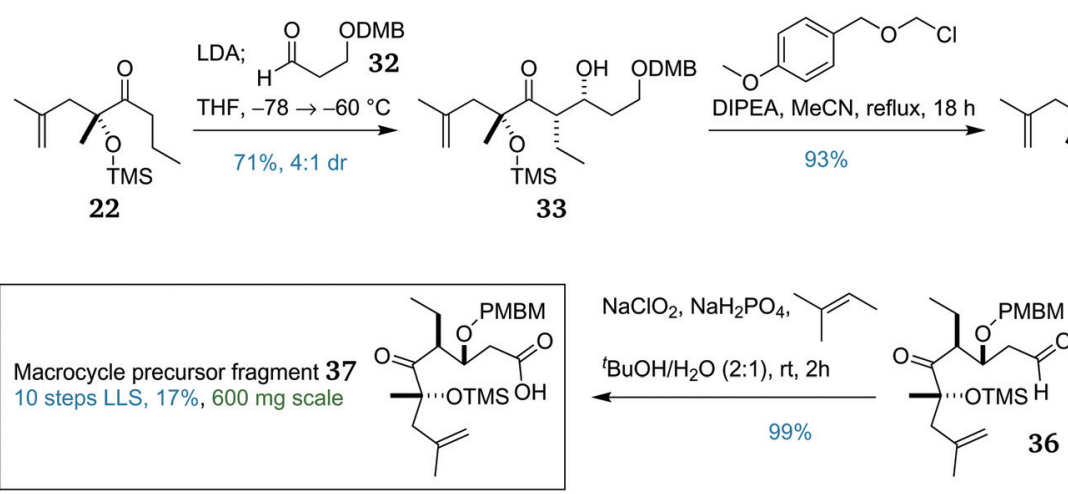

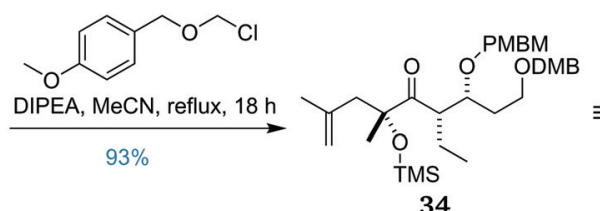

34

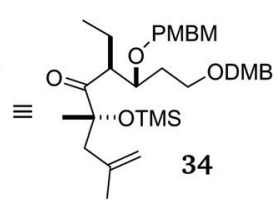

$\mathrm{DDQ}, 0^{\circ} \mathrm{C}, 2 \mathrm{~h}$ $\mathrm{CH}_{2} \mathrm{Cl}_{2} / \mathrm{pH} 7$ buffer $(9: 1), 91 \%$

Scheme 4 Completion of the synthesis of macrocycle precursor fragment 37. 
$17 \%$. This represents an average of $84 \%$ per step, and the scalability of the route allowed $600 \mathrm{mg}$ to be prepared, providing ample material for development of the endgame.

\section{Fragment union and endgame}

With key fragments 8 and 37 in hand, the stage was set to explore the pivotal fragment union sequence. The retrosynthetic plan (Scheme 1) had left the ordering of these steps flexible. However, preliminary investigations had indicated that an efficient and stereoselective cross metathesis to give the trisubstituted $8 E$ alkene was unlikely to succeed, so we proceeded with exploring the esterification/RCM sequence. The fragments 37 and $\mathbf{8}$ were coupled together under standard Yamaguchi conditions ${ }^{48}$ to afford ester 38 in an excellent yield of $99 \%$, enabling investigation of the "do-or-die" macrocycleforming RCM step (Scheme 5).

The first attempt at cyclisation of $\mathbf{3 8}$ using Grubbs secondgeneration catalyst (G-II) ${ }^{49,50}$ in refluxing degassed $\mathrm{CH}_{2} \mathrm{Cl}_{2}$ provided only the undesired dimer $\mathbf{4 0}$ in quantitative yield (Table 1, entry 1). Increasing the catalyst loading to $50 \mathrm{~mol} \%$ (entry 2) or the use of higher-boiling solvents (entries 3 and 4) failed to provide any macrocyclic product, in each case returning only dimer 40. As an additional measure, a solution of catalyst was added portionwise over the initial few hours of the reaction to reduce catalyst decomposition, although the colour change from purple to brown was observed within $30 \mathrm{~min}$ of each addition, indicating rapid catalyst degradation. In light of these failures, we switched to the more reactive HoveydaGrubbs second-generation catalyst (HG-II). ${ }^{51,52}$ Gratifyingly, an initial RCM reaction of 38 , overnight in refluxing toluene, afforded an inseparable 1:1 mixture of dimer $\mathbf{4 0}$ and desired macrocycle 39 (entry 5). Increasing the catalyst loading to $40 \mathrm{~mol} \%$ and the reaction time to $72 \mathrm{~h}$ increased the product: dimer ratio to $1.5: 1$ (entry 6). In an attempt to increase the reaction rate and yield, the solvent was changed to $o$-xylene to allow reflux at a higher temperature. Surprisingly, this led to a reduction in the product: dimer ratio to $1.2: 1$ (entry 9), indicating that there was a 'sweet spot' temperature at around $110-140{ }^{\circ} \mathrm{C}$, below which the reaction is prohibitively slow and above which catalyst decomposition occurs.

By this point, a mechanistic hypothesis was proposed whereby the terminal alkene present in $\mathbf{3 8}$ rapidly dimerises. The ruthenium catalyst can then reinsert into the new disubstituted alkene in this dimer and can then undergo one of two processes. In most cases, this undergoes an intermolecular reaction with another molecule of dimer $\mathbf{4 0}$ to rever-
Table 1 Screening of ring-closing metathesis conditions for the formation of macrocycle 39 from precursor 38 (G-II = Grubbs secondgeneration catalyst, HG-II = Hoveyda-Grubbs second-generation catalyst)

\begin{tabular}{llllll}
\hline Entry & Cat. (mol\%) & Solvent (mM) & Temp. & Time & $39: 40$ \\
\hline 1 & G-II (20) & $\mathrm{CH}_{2} \mathrm{Cl}_{2}(5)$ & $40^{\circ} \mathrm{C}$ & $18 \mathrm{~h}$ & $0: 1$ \\
2 & G-II (50) & $\mathrm{CH}_{2} \mathrm{Cl}_{2}(10)$ & $40^{\circ} \mathrm{C}$ & $18 \mathrm{~h}$ & $0: 1$ \\
3 & G-II (20) & Benzene (1) & $80^{\circ} \mathrm{C}$ & $18 \mathrm{~h}$ & $0: 1$ \\
4 & G-II (20) & Toluene (1) & $110^{\circ} \mathrm{C}$ & $18 \mathrm{~h}$ & $0: 1$ \\
5 & HG-II (20) & Toluene (1) & $110^{\circ} \mathrm{C}$ & $18 \mathrm{~h}$ & $1: 1$ \\
6 & HG-II (40) & Toluene (1) & $110^{\circ} \mathrm{C}$ & $72 \mathrm{~h}$ & $1.5: 1$ \\
7 & HG-II (40) & Toluene (10) & $110^{\circ} \mathrm{C}$ & $72 \mathrm{~h}$ & $1.3: 1$ \\
8 & HG-II (40) & Toluene (0.25) & $110^{\circ} \mathrm{C}$ & $72 \mathrm{~h}$ & $2: 1$ \\
9 & HG-II (40) & Xylene (0.25) & $145^{\circ} \mathrm{C}$ & $72 \mathrm{~h}$ & $1.2: 1$ \\
10 & HG-II (40) & Toluene (1) & $110^{\circ} \mathrm{C}$ & $7 \mathrm{~d}$ & $3: 1$
\end{tabular}

sibly return to the dimer. Much more slowly, but irreversibly, this ruthenium carbene can undergo an intramolecular reaction with the 1,1-disubstituted alkene to afford desired macrocycle 39. To help encourage the slow, irreversible RCM step, the duration of the reaction was increased to seven days, increasing the product: dimer ratio to $3: 1$ and providing the desired product with an improved yield of $75 \%$ (entry 10). To our knowledge, this challenging and remarkable transformation is the most complex example of an RCM reaction to form a trisubstituted alkene in a medium-sized ring. ${ }^{23}$

Having prepared an abundant supply of advanced intermediate 39, the removal of both PMB-containing protecting groups was accomplished by treatment with DDQ, affording diol 41 in 94\% yield (Scheme 6). Chemoselective oxidation of the primary alcohol at $\mathrm{C}_{21}$ in the presence of the $\mathrm{C}_{3}$ secondary alcohol was achieved using a TEMPO/BAIB oxidation ${ }^{53,54}$ which afforded aldehyde $\mathbf{4 2}$ in $90 \%$ yield. Subsequently, chemoselective addition of ethylmagnesium bromide to the newly formed aldehyde in the presence of the $\mathrm{C}_{5}$ ketone proceeded smoothly, affording an inseparable epimeric mixture of 43 in $91 \%$ yield, with no observable attack at the ketone.

Having completed the full carbon backbone of the actinoallolides, our attention now turned to the final two steps of oxidation and global deprotection. Oxidation of both secondary alcohols at $\mathrm{C}_{3}$ and $\mathrm{C}_{21}$ was initially attempted under Swern conditions, however, this was found to be unreliable. Alternatively, the oxidation was performed using the milder Dess-Martin periodinane. ${ }^{55}$ Double oxidation under these conditions was found to be clean and reliable, providing triketone 44 in 99\% yield.

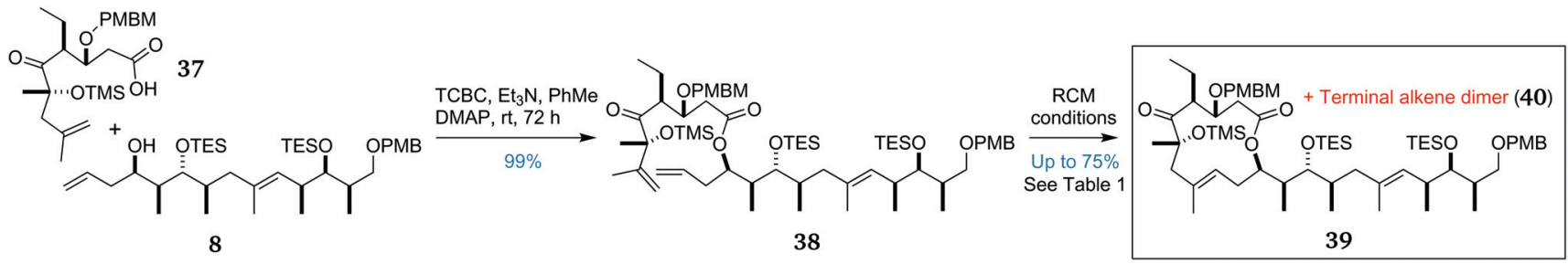

Scheme 5 Fragment union and RCM-mediated formation of the 12-membered macrocycle 39. 

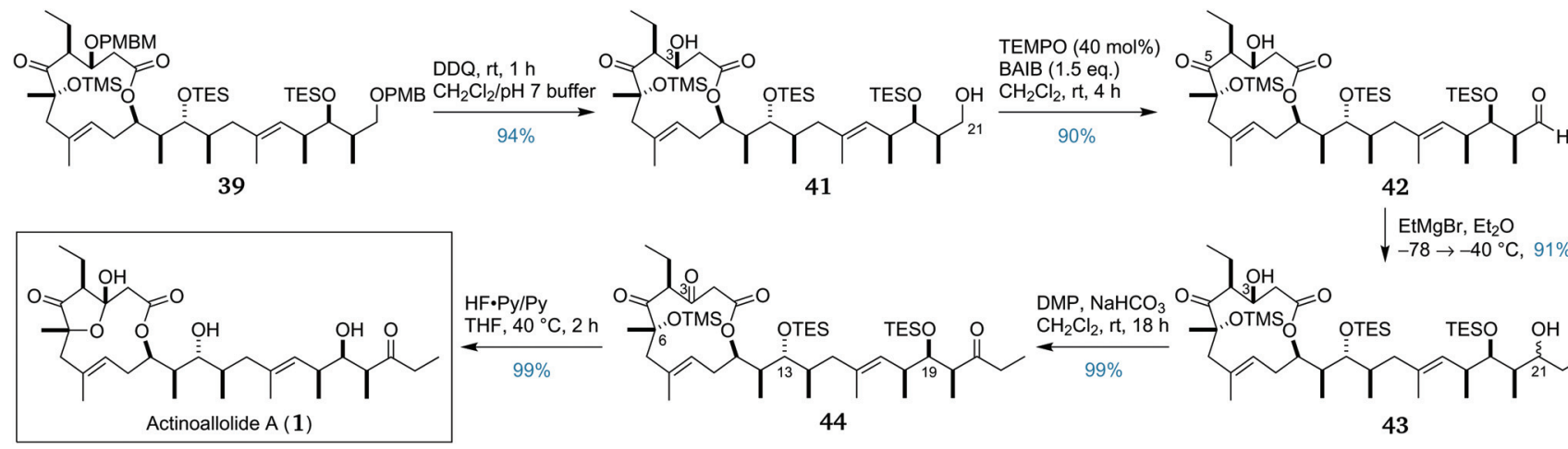

$\mathrm{EtMgBr}, \mathrm{Et}_{2} \mathrm{O}$

V $-78 \rightarrow-40^{\circ} \mathrm{C}, 91 \%$

Scheme 6 Endgame and completion of the total synthesis of actinoallolide A (1).

The only obstacle now remaining was the final deprotection. The conditions should ensure complete cleavage of the $\mathrm{C}_{6}$ TMS ether and the $\mathrm{C}_{13}$ and $\mathrm{C}_{19}$ TES ethers, while also forming the transannular hemiacetal. The conditions were also required to be sufficiently mild to avoid any possible deleterious side reactions. Preliminary experience with the lability of the hemiacetal motif led to the hypothesis that actinoallolides C-E may be artefacts of the elaborate chromatographic isolation process. Consequently, we were particularly cautious to avoid elimination across the $\mathrm{C}_{3}-\mathrm{C}_{4}$ bond and transesterification of the macrolactone in 44. Initial attempts using TBAF were unsuccessful, leading either to incomplete deprotection or degradation. Pleasingly, acidic fluorous deprotection conditions proved successful, with treatment of $\mathbf{4 4}$ with a $1: 3$ mixture of HF.py/py with warming to $40{ }^{\circ} \mathrm{C}$ effecting quantitative conversion to (+)-actinoallolide A (1). The resulting synthetic actinoallolide A possessed comparable specific rotation and identical NMR spectra to those of the natural product (see the ESI $\dagger$ ).

The total synthesis of (+)-actinoallolide A (1) was thus achieved in 20 linear steps from $(R)$-Roche ester (14) with an overall yield of $8 \%$, representing an average yield of $88 \%$ per step. Importantly, this first total synthesis gave an orthogonal validation of the full 3D structure and absolute configuration of actinoallolide A and will enable further biological investigations of this highly potent family of natural products.

\section{Conversion of actinoallolide A to actinoallolides B-E}

We next sought to access the other four congeners, actinoallolides B-E (2-5), by replication of the conversion protocols in the isolation paper (Scheme 7). ${ }^{18}$ Actinoallolide A (1) underwent a 1,3-syn hydroxyl-directed reduction using triethylborane followed by sodium borohydride to afford actinoallolide B (2) in 93\% yield and as a single diastereomer. Subsequent treatment of actinoallolide B (2) with trifluoroacetic acid caused dehydration of the cyclic hemiacetal to provide actinoallolide D (4) in $99 \%$ yield.

During the initial purification of actinoallolide A using silica chromatography, some minor by-product formation was observed. Using alumina instead, attempted purification of actinoallolide A led to conversion to a $1: 1$ mixture of actinoal-

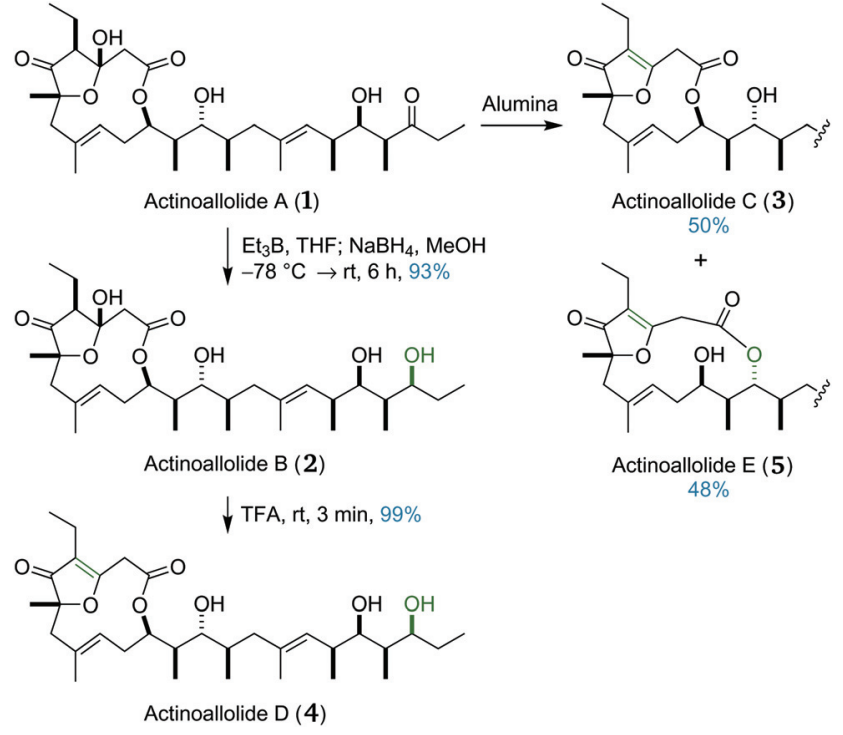

Scheme 7 Conversion of actinoallolide A (1) to actinoallolides B-E (2-5).

lolide C (3) and actinoallolide E (5). This unexpected result indicated the instability of the cyclic hemiacetal moiety to basic conditions, perhaps explaining why global deprotection was unsuccessful under basic fluorous conditions. This spontaneous conversion of actinoallolide A (1) to actinoallolides C (3) and E (5) on alumina also provides evidence that these compounds are isolation artefacts rather than genuine natural products. This serendipitous result completed our synthesis of all five members of the actinoallolides.

Based on the assumption that actinoallolides $\mathrm{C}$ and $\mathrm{E}$ are isolation artefacts, it is likely that actinoallolide D is also an artefact, arising from dehydration of actinoallolide B. This would leave actinoallolides A and B as the only true natural products, differing only in the oxidation level at $\mathrm{C}_{21}$. In examining the proposed biosynthesis of actinoallolide $\mathrm{A},{ }^{20}$ the authors had identified an inactive ketoreductase domain (KR1) in the polyketide synthase responsible for the biosynthesis of actinoallolide A. They commented that "although KR1 has catalytic amino acids and the NADPH binding motif, it seems 
to be inactive, as predicted from the structure of actinoallolide A that has a ketone at $\mathrm{C}_{21}$ ". If, rather than being inactive, the KR1 domain were instead partially active, this identified polyketide synthase would be able to generate both actinoallolides $\mathrm{A}$ and $\mathrm{B}$. When the KR1 domain performs the $\mathrm{C}_{21}$ reduction,
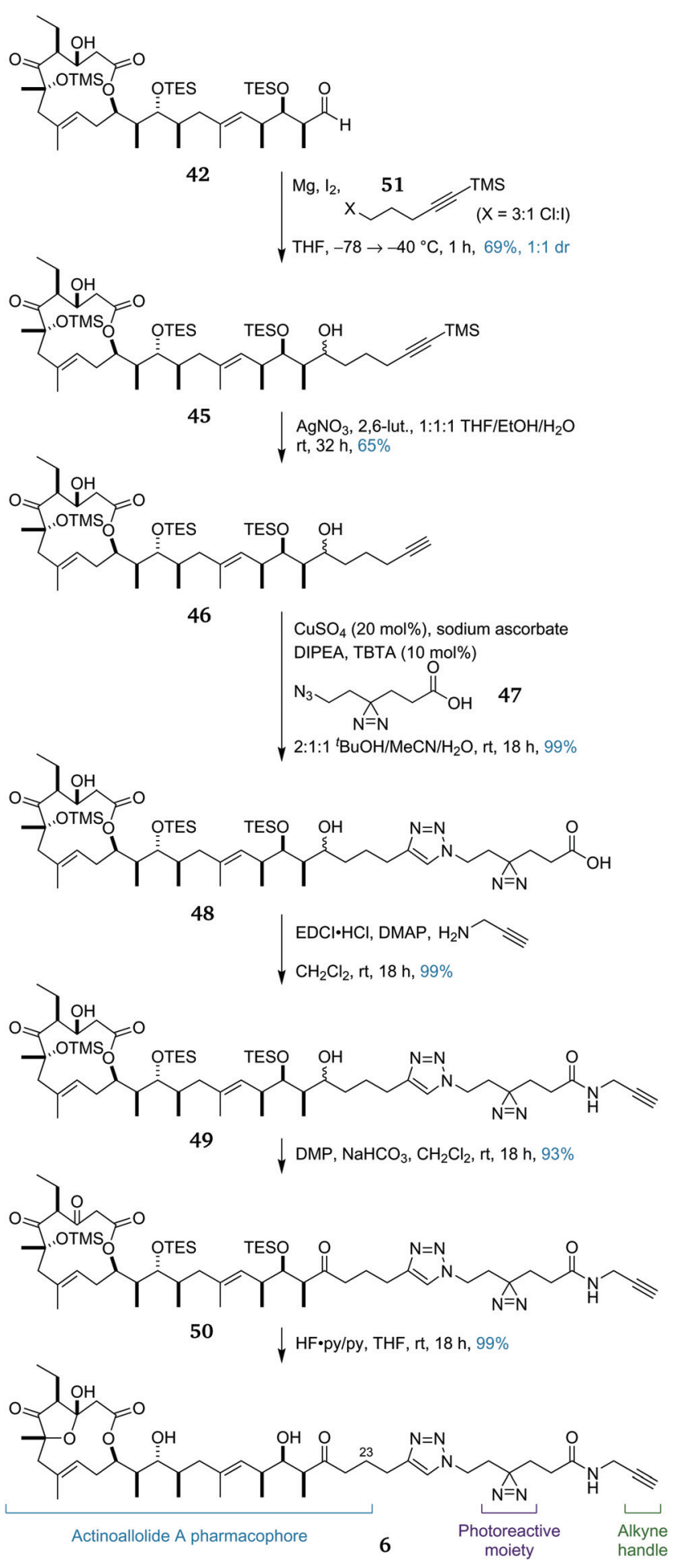

Scheme 8 Diversion of advanced intermediate 42 towards the synthesis of photoaffinity probe analogue 6 of actinoallolide A. actinoallolide $\mathrm{B}$, containing the $\mathrm{C}_{21}$ alcohol, is produced, and when transfer to module 2 occurs before the reduction can take place, the product is instead actinoallolide $\mathrm{A}$, with the unreduced $\mathrm{C}_{21}$ ketone. On inspection of the amino acid sequence of the KR1 domain, it possesses both sets of characteristic amino acids which determine whether reduction affords the $\mathrm{L}^{-}$or $\mathrm{D}$-configuration, potentially explaining its partial inactivity.

\section{Synthesis of an actinoallolide A photoaffinity probe analogue}

Having achieved a practical, convergent and high-yielding total synthesis of the actinoallolides, we moved onto the design and synthesis of a photoaffinity probe analogue (Scheme 8) to identify their biological target. In order to minimally perturb the structure of the native natural product, the photoaffinity probe 6 was chosen to extend from the $\mathrm{C}_{23}$ terminus. ${ }^{56}$ The photoreactive moiety is a diazirine, decomposing to a highlyreactive carbene upon irradiation at $365 \mathrm{~nm}$, facilitating crosslinking to the bound target protein. The functional handle is a terminal alkyne, allowing the facile conjugation of any desired reporter tag using a copper-catalysed azide-alkyne cycloaddition. $^{25}$

The synthesis of probe $\mathbf{6}$ began by intercepting the advanced intermediate 42. In the total synthesis, addition of an ethyl group was required to complete the actinoallolide carbon skeleton. Instead, the addition of the Grignard reagent derived from halide 45 afforded diol 46 in 69\% yield as an inconsequential 1:1 mixture of diastereomers. Chemoselective removal of the alkynyl TMS group was achieved by treatment with $\mathrm{AgNO}_{3}$ and gave 47 in $65 \%$ yield. Next, a copper-catalysed azide-alkyne cycloaddition between alkyne 47 and linker fragment 48 (prepared from 1,4-cylohexanedione in eight steps $)^{57}$ proceeded to afford triazole 49 (99\%).

The EDCI/DMAP-mediated amidation of 49 with propargylamine proceeded smoothly, affording amide $\mathbf{5 0}$ in $99 \%$ yield. This amide then underwent a double DMP-mediated oxidation to afford triketone $\mathbf{5 1}$ (93\%). Finally, global deprotection under acidic fluorous conditions proceeded with an excellent yield of 99\%, cleaving all three silyl ethers and inducing formation of the transannular five-membered hemiacetal to form photoaffinity probe $\mathbf{6}$. To date, this route has provided $26 \mathrm{mg}$ of $\mathbf{6}$, sufficient material for the required photoaffinity experiments. In preliminary studies, the biological activities of synthetic actinoallolide A (1) and photoaffinity probe $\mathbf{6}$ have been confirmed and photoaffinity studies are currently ongoing.

\section{Conclusions}

This account details the first total synthesis of the actinoallolides, a family of polyketide natural products prized for their promising anti-trypanosomal properties. ${ }^{58}$ This feat has been accomplished in a scalable, high-yielding and stereoselective fashion, giving actinoallolide A (1) in 20 linear steps from $(R)$ Roche ester (14) with an overall yield of $8 \%$. This was achieved in a highly convergent manner, utilising two fragments 8 and 
37 of similar complexity, which were obtained in 13 and 10 steps respectively. The stubbornness of $\mathrm{C}_{3}$ manipulation to standard 'PMB transposition' conditions was frustrating and necessitated modifications to the original strategy. Fortunately, the judicious choice of alternative protecting groups allowed us to overcome this obstacle.

Fragment union was accomplished by esterification followed by a highly challenging ring-closing metathesis, gratifyingly forming the macrocyclic trisubstituted alkene as a single geometrical isomer. This "do-or-die" transformation required significant optimisation and is the most complex example of its kind. A high-yielding endgame finally afforded actinoallolide A. Actinoallolide A was then converted to actinoallolides B-E, completing the total synthesis of the entire natural product family. The alumina-mediated conversion of actinoallolide A to actinoallolides $\mathrm{C}$ and $\mathrm{E}$ provides evidence that actinoallolides $\mathrm{C}-\mathrm{E}$ are isolation artefacts, with actinoallolides $\mathrm{A}$ and $\mathrm{B}$ as the only true natural products. On review of the biosynthetic data for the actinoallolides, a partially active ketoreductase domain in the actinoallolide A polyketide synthase has been postulated to be responsible for generating these two congeneric natural products.

With the secondary aim of probing how actinoallolide A exerts its highly specific biological effects, this route was diverted to give $\mathbf{6}$ as a designed photoaffinity probe. This was achieved in six steps from advanced intermediate 42. Ongoing work is focused on using 6 in various assays with the aim of identifying the biological target of actinoallolide A. Identification of this target and knowledge of the mechanism of action may allow the generation of simplified, more synthetically tractable or more potent actinoallolide analogues in the ongoing effort to develop new, effective medicinal agents to treat neglected tropical diseases.

\section{Conflicts of interest}

There are no conflicts to declare.

\section{Acknowledgements}

We thank the Herchel Smith Fund (studentship to M. J. A.), Dr Rachel J. Porter and Dr Simon Williams for assistance, and Thomas Hayhow (AstraZeneca) for his interest. We are grateful to Professor Terry Smith and Dr Gordon Florence at the University of St Andrews for performing the preliminary bioactivity assays. We also acknowledge the National Mass Spectrometry Facility at Swansea University.

\section{References}

1 C. Mathers, G. Stevens, W. R. Mahanani, J. Ho, D. M. Fat and D. Hogan, Global Health Estimates 2015: Deaths by Cause, Age, Sex, by Country and by Region, 2000-2015, World health organization technical report, 2016.
2 P. J. Hotez and A. Kamath, PLoS Neglected Trop. Dis., 2009, 3, e412.

3 P. Büscher, G. Cecchi, V. Jamonneau and G. Priotto, Lancet, 2017, 390, 2397-2409.

4 R. Brun, J. Blum, F. Chappuis and C. Burri, Lancet, 2010, 375, 148-159.

5 Trypanosomes and Trypanosomiasis, ed. S. Magez and M. Radwanska, Springer-Verlag, Wien, 2014.

6 J. Bermudez, C. Davies, A. Simonazzi, J. P. Real and S. Palma, Acta Trop., 2016, 156, 1-16.

7 J. A. Pérez-Molina and I. Molina, Lancet, 2018, 391, 82-94.

8 C. Bern, N. Engl. J. Med., 2015, 373, 456-466.

9 J. D. Stanaway and G. Roth, Global Heart, 2015, 10, 139144.

10 P. J. Hotez, E. Dumonteil, L. Woc-Colburn, J. A. Serpa, S. Bezek, M. S. Edwards, C. J. Hallmark, L. W. Musselwhite, B. J. Flink and M. E. Bottazzi, PLoS Neglected Trop. Dis., 2012, 6, e1498.

11 WHO Disease burden and mortality estimates, http://www. who.int/healthinfo/global_burden_disease/estimates/en/, (accessed September 2020).

12 D. J. Barry and R. McCulloch, Adv. Parasitol., 2001, 49, 1-70.

13 M. C. Field, D. Horn, A. H. Fairlamb, M. A. J. Ferguson, D. W. Gray, K. D. Read, M. De Rycker, L. S. Torrie, P. G. Wyatt, S. Wyllie and I. H. Gilbert, Nat. Rev. Microbiol., 2017, 15, 217-231.

14 P. E. Cockram and T. K. Smith, J. Nat. Prod., 2018, 81, 2138-2154.

15 A. Strelkauskas, A. Edwards, B. Fahnert, G. Pryor and J. Strelkauskas, Microbiology: A Clinical Approach, Garland Science, 2015.

16 A. R. Renslo and J. H. McKerrow, Nat. Chem. Biol., 2006, 2, 701-710.

17 L. Paloque, A. Triastuti, G. Bourdy and M. Haddad, Natural Antimicrobial Agents, Springer International Publishing, Cham, 2018, pp. 215-245.

18 Y. Inahashi, M. Iwatsuki, A. Ishiyama, A. Matsumoto, T. Hirose, J. Oshita, T. Sunazuka, W. Panbangred, Y. Takahashi, M. Kaiser, K. Otoguro and S. Omura, Org. Lett., 2015, 17, 864-867.

19 B. L. Roth, The Serotonin Receptors: From Molecular Pharmacology to Human Therapeutics, Springer Science and Business Media, 2008.

20 Y. Inahashi, T. Shiraishi, A. Také, A. Matsumoto, Y. Takahashi, S. Omura, T. Kuzuyama and T. Nakashima, J. Antibiot., 2018, 71, 749-752.

21 J. Oshita, Y. Noguchi, A. Watanabe, G. Sennari, S. Sato, T. Hirose, D. Oikawa, Y. Inahashi, M. Iwatsuki, A. Ishiyama, S. Omura and T. Sunazuka, Tetrahedron Lett., 2016, 57, 357-360.

22 For a preliminary communication, see: M. J. Anketell, T. M. Sharrock and I. Paterson, Angew. Chem., 2020, 59, 1572-1576.

23 C. Lecourt, S. Dhambri, L. Allievi, Y. Sanogo, N. Zeghbib, R. B. Othman, M.-I. Lannou, G. Sorin and J. Ardisson, Nat. Prod. Rep., 2018, 35, 105-124. 
24 E. Smith and I. Collins, Future Med. Chem., 2015, 7, 159183.

25 L. B. Tulloch, S. K. Menzies, A. L. Fraser, E. R. Gould, E. F. King, M. K. Zacharova, G. J. Florence and T. K. Smith, PLoS Neglected Trop. Dis., 2017, 11, e0005886.

26 I. Paterson, G. J. Florence, K. Gerlach, J. P. Scott and N. Sereinig, J. Am. Chem. Soc., 2001, 123, 9535-9544.

27 I. Paterson, S. J. Fink, L. Y. W. Lee, S. J. Atkinson and S. B. Blakey, Org. Lett., 2013, 15, 3118-3121.

28 I. Paterson and R. D. Tillyer, Tetrahedron Lett., 1992, 33, 4233-4236.

29 J. G. Solsona, J. Nebot, P. Romea and F. Urpí, J. Org. Chem., 2005, 70, 6533-6536.

30 J. Esteve, S. Matas, M. Pellicena, J. Velasco, P. Romea, F. Urpí and M. Font-Bardia, Eur. J. Org. Chem., 2010, 31463151.

31 R. E. Ireland and R. H. Mueller, J. Am. Chem. Soc., 1972, 94, 5897-5898.

32 I. Paterson and A. N. Hulme, J. Org. Chem., 1995, 60, 32883300.

33 I. Paterson and D. J. Wallace, Tetrahedron Lett., 1994, 35, 9087-9090.

34 I. Paterson, D. J. Wallace and C. J. Cowden, Synthesis, 1998, 639-652.

35 J. H. Lee, Tetrahedron, 2020, 76, 131351.

36 A. B. Smith, T. J. Beauchamp, M. J. LaMarche, M. D. Kaufman, Y. Qiu, H. Arimoto, D. R. Jones and K. Kobayashi, J. Am. Chem. Soc., 2000, 122, 8654-8664.

37 R. Nagase, Y. Oguni, T. Misaki and Y. Tanabe, Synthesis, 2006, 3915-3917.

38 D. Seebach, R. Naef and G. Calderari, Tetrahedron, 1984, 40, 1313-1324.

39 J. S. Kingsbury and E. J. Corey, J. Am. Chem. Soc., 2005, 127, 13813-13815.

40 G. Ehrlich, J. Hassfeld, U. Eggert and M. Kalesse, J. Am. Chem. Soc., 2006, 128, 14038-14039.

41 R. Johansson and B. Samuelsson, J. Chem. Soc., Perkin Trans. 1, 1984, 2371-2374.

42 D. A. Evans and A. H. Hoveyda, J. Am. Chem. Soc., 1990, 112, 6447-6449.

43 D. A. Evans, K. T. Chapman and E. M. Carreira, J. Am. Chem. Soc., 1988, 110, 3560-3578.
44 A. P. Kozikowski and J.-P. Wu, Tetrahedron Lett., 1987, 28, 5125-5128.

45 Y. Oikawa, T. Tanaka, K. Horita, T. Yoshioka and O. Yonemitsu, Tetrahedron Lett., 1984, 25, 5393-5396.

46 D. A. Evans, P. H. Carter, E. M. Carreira, A. B. Charette, J. A. Prunet and M. Lautens, J. Am. Chem. Soc., 1999, 121, 7540-7552.

47 O. P. Anderson, A. G. Barrett, J. J. Edmunds, S.-I. Hachiya, J. A. Hendrix, K. Horita, J. W. Malecha, C. J. Parkinson and A. VanSickle, Can. J. Chem., 2001, 79, 1562-1592.

48 J. Inanaga, K. Hirata, H. Saeki, T. Katsuki and M. Yamaguchi, Bull. Chem. Soc. Jpn., 1979, 52, 1989-1993.

49 A. K. Chatterjee and R. H. Grubbs, Org. Lett., 1999, 1, 17511753.

50 O. M. Ogba, N. C. Warner, D. J. O'Leary and R. H. Grubbs, Chem. Soc. Rev., 2018, 47, 4510-4544.

51 S. B. Garber, J. S. Kingsbury, B. L. Gray and A. H. Hoveyda, J. Am. Chem. Soc., 2000, 122, 8168-8179.

52 S. Gessler, S. Randl and S. Blechert, Tetrahedron Lett., 2000, 41, 9973-9976.

53 A. De Mico, R. Margarita, L. Parlanti, A. Vescovi and G. Piancatelli, J. Org. Chem., 1997, 62, 6974-6977.

54 A. E. J. d. Nooy, A. C. Besemer and H. v. Bekkum, Synthesis, 1996, 1153-1176.

55 D. B. Dess and J. C. Martin, J. Org. Chem., 1983, 48, 41554156.

56 S. Ho, D. L. Sackett and J. L. Leighton, J. Am. Chem. Soc., 2015, 137, 14047-14050.

57 J. D. Mortison, M. Schenone, J. A. Myers, Z. Zhang, L. Chen, C. Ciarlo, E. Comer, S. K. Natchiar, S. A. Carr, B. P. Klaholz and A. G. Myers, Cell Chem. Biol., 2018, 25, 1506-1518.

58 For recent work by our group on other complex bioactive macrolides, see: (a) A. W. Phillips, M. J. Anketell, T. Balan, N. Y. S. Lam, S. Williams and I. Paterson, Org. Biomol. Chem., 2018, 16, 8286-8291; (b) S. Williams, J. Jin, S. B. J. Kan, M. Li, L. J. Gibson and I. Paterson, Angew. Chem., Int. Ed., 2017, 56, 645-649; (c) N. Anžiček, S. Williams, M. P. Housden and I. Paterson, Org. Biomol. Chem., 2018, 16, 1343-1350; (d) N. Y. S. Lam, G. Muir, V. R. Challa, R. Britton and I. Paterson, Chem. Commun., 2019, 55, 9717-9720. 